\title{
Relationship and Influence of Mathematical Theory and Mathematical Application under Horizon of College Mathematical Teaching
}

\author{
Dalu Wang \\ Yellow River Conservancy Technical Institute, Kaifeng 475004, China
}

Keywords: College mathematics; Mathematical theory; Mathematical application

\begin{abstract}
This paper explains the relationship and influence of mathematical theory and application in college mathematical teaching, and proposes that the mathematical theory is not only the key part of college mathematical teaching, but also the important basis for completing college mathematical teaching program. In order to realize the improvement of mathematical theory teaching, it is required to make connection with mathematical application. Under general condition, the mathematical application is divided into two parts, that is, external and internal application; however, in college mathematical teaching process, the category and layer division shall be made in the cultivation of students' mathematical application ability.
\end{abstract}

\section{Introduction}

For a long time, the mathematical education of Chinese institutions of higher learning only pays attention to education on mathematical basic theory and learning skills, but ignores the important significance of mathematical application. Therefore, this causes a situation that the college students have weak ability of thinking in practice and can't effectively make use of applied mathematics to solve practical problems. Although the government department has proposed reform suggestion, the reform content only focuses on textbooks and teaching methods, but doesn't obtain considerable effect in enhancing students' mathematical application ability. Based on this, this paper starts from the actual situation of college mathematical teaching to focus on analysis the relationship existing between mathematical theory and mathematical application for the purpose of enhance college attention paid to mathematical application ability and carrying out cultivating college students' mathematical application ability.

\section{Foundation of mathematical education - mastery of basic mathematical theory and methods}

As for mathematical education, one of the important purposes is to make students know and understand basic mathematical theory and freely use basic mathematical methods. If we can't lay a good foundation of basic mathematical theory and methods, we will be unable to realize the purpose of advocating mathematical innovative thinking, enhancing application ability, and improving overall quality. Therefore, the most critical two objectives of college mathematical education are to realize the mathematical application and improve innovative ability.

The current situation of mathematical theory education is worrying.

Although the first response at mentioning the mathematical education reform is that we have input large quantity of time and energy in mathematical theory education and we are not aware of cultivating students' mathematical application ability, do the teaching status and teaching effect of college mathematical theory reach the scheduled standard? Through research and analysis, the author finds that many students majoring in science will not or not actively devote themselves into mathematical learning, and even be feared about mathematical theory due to the influence of graduation, employment, and pragmatism. ${ }^{[1]}$ Besides, while designing graduation thesis, some students always make a detour on topics closely related to mathematical theory. Although they have smoothly passed mathematics course examination, it is still unable to guarantee that they have had the ability to systematically master basic mathematical theory and method. The author thinks that it is required to not carry out continuous trial on mathematical application practice at undergraduate 
course as for cultivating professional mathematical talents. This is because it becomes a behavior of attending to trifles and neglecting the essentials if the undergraduate students refuse to do basic mathematical projects. Once the college students majoring in mathematics give no key learning to the fundamental knowledge, that is, basic mathematical theory, it will be hard for them to obtain excellent result in the future mathematical theory or make progress in mathematical application. This is because the undergraduate students will have no competition with other talents under the condition of no advantage of engineering major and mathematics major. Similarly, if the students majoring in engineering have been confused in learning basic mathematical theory, there will be negative influence on his learning process of professional knowledge, which restricts the improvement of their professional ability ${ }^{[2]}$. Nevertheless, the mathematical theory and methods can be seen in natural science and engineering technology, and some engineering technical personnel have clearly showed that their knowledge of mathematical theory is so insufficient that they can't make further progress in their profession.

\section{The mathematical theory is the foundation of mathematical application}

The range and degree of mathematical theory that the personnel who engage in science and technology work master are closely related to their creative ability, and the historical practice experience also proves this statement. The physicist Dirac is a very typical example; he made use of his mathematical theoretical knowledge to handle many kinds of wave functions, and used symmetric wave functions to describe that boson conformed to Bose-Einstein statistics rules, and also used anti-symmetric wave function to describe that the fermion conformed to Fermi-Dirac statistics rules ${ }^{[3]}$. As for physical science, this was a milestone-type development. However, although Dirac got major discovery through this experiment, there lacked of systematic mathematical training; the experimental result was not improved to theoretical level, and no foreseeing was proposed in terms of theory. Later, Maxwell utilized his mathematical knowledge to improve Faraday's qualitative experiment to quantitative theoretical level, and Maxwell differential equation was successfully established. Except that, there are many practical cases which can show that the systematic mathematical theory education is of important significance, and the most critical link in current mathematical education reform is to improve the teaching level of mathematical theory; otherwise it is just an empty talk as for improving mathematical application ability.

\section{Relationship and influence of mathematical theory and application}

As a whole, the implementation objective of college mathematical education is to improve college students' mathematical literary which involves theory, thinking method, and application awareness. People summarize the source of development of mathematical theory and methods as two points: one is to solve practical problems in science, technology, and production practice, and the other is to be driven by the rules of development of mathematical theory. These two sources are actually mathematical application. Then, we will discuss the function of these two sources on teaching of mathematical theory.

\section{People's viewpoints on the relationship between mathematical theory and application}

One senior international academician once said that the wide scholars could intuitively know that the mathematics was a kind of language which could express the scientific thoughts in a detailed and accurate way, and could realize that the science could influence the progress of mathematical thoughts to a great degree in calculus. Moreover, he also proposed that the mathematics could correctly and reasonably answer many critical scientific problems. The professor Wang Liyun showed that people should continuously make reflection on the practical case of those important practical mathematical thoughts, methods, and application. ${ }^{[5]}$ In the process of teaching mathematical theory and methods, if the teachers can't show perfect and comprehensive "logic chain" in classroom teaching, can't clearly explain production, evolution and development history of relevant concepts and methods, and can't show a specific and vivid mathematical thinking process and actual application process of mathematical methods to students, the students will be unable to realize the expected learning objective from this learning process, and the whole teaching process becomes dull. Besides, the professor Tan Yingya also clearly showed that the college textbooks at current stage were complete mathematical content from the starting part, and rarely mentioned the source and 
applicable range of this part of content; in this way, the students couldn't know the origin and development of those contents, thus many students thought that the essence of mathematics was just a series of assumption, thinking, and problem solving. Therefore, it is very hard to use a kind of teaching method to make students know the reason and influence factors for mathematical development and master the essence of mathematical theory.

\section{Function of mathematical application on mathematical theory}

The historic practice shows that the applied mathematics and pure mathematics were linked together before 1870s; in other words, the mathematics at current stage realizes its progress and development almost by virtue of actual application. Through deep research, it can be found that the purpose of production and progress of many kinds of mathematical theory is to solve the actual difficulty faced under current stage, and the appearance of calculus explains this point well. The main body of college mathematics appeared before 1870s; however, could people truly realize long-term and stable development of mathematical theory under the premise of ignoring the mathematical application? This answer is certainly negative; through linking with the source of mathematical application, it is able to make students correctly know how the mathematics determines the essence and property of problems and carries out a reasonable handling, and then realize coming from reality but being higher than reality. To know the abstractness of mathematics is not to make problems become complicated, but simplify the problems so as to effectively apply final result into the practice. Actually, this is the thought and great mathematical function of solving the actual problems via mathematics, and this function attracts people's wide attention under current period. If people can guide students to this realm, they will certainly see the great attraction of mathematics, know the connotation and method of mathematics from perspective of essence, learn to carry out deep research on the nature existing under abstract appearance, and then enhance their awareness of mathematical application. In this way, we can say that the mathematical theory teaching is integral.

In a word, while we divide the mathematical application into external and internal application, we shall also closely link mathematical theoretical education with improvement of application ability together. ${ }^{[6]}$ Once people only pay attention to the theoretical teaching and ignore the importance of application ability, the self-contradiction will happen, and they will neither get an essential understanding of mathematical theory and method nor realize systematic and overall mathematical theory teaching.

\section{Highest objective of college mathematical education - cultivating overall mathematical application ability}

The overall mathematical application mentioned here doesn't specifically refers to the practical application of mathematics, for it is one-sided. As the American mathematician Jenifor Mark points out, the mathematics application shall be divided into two types, that is, internal and external application, in order to realize the final objective of mathematical education, that is, improving students' mathematical application ability, we must carry out this division. ${ }^{[7]}$ However, the difference in training object and training purpose also means that we shall carry out category and layer division as for cultivating mathematical application ability. Prior to division, we shall specify one point, that is, the training objective of mathematical application ability of college mathematical education is different from solving partial practical problems in secondary education. Furthermore, we shall pay special attention to cultivating college mathematical application ability and carry out layer and objective division. For example, the college students who major in engineering or intend to select practical mathematical application work in the future shall focus on cultivating their external mathematical application ability, and the college students who intend to select mathematical research work in the future shall focus on cultivating their internal mathematical application ability.

While understanding the mathematical theory and application, some people hold a kind of superficial or one-sided viewpoints and propose that the mathematics subject is driven by practical problems in long-time development process, thus the final objective of mathematical education becomes mastering the ability of utilizing the mathematics to solve actual problems. The author 
thinks that this opinion is one-sided in theoretical layer and practical layer, and it doesn't conform to the meaning of mathematics subject and its historical development features. This opinion weakens the function of mathematical education, restricts cultivating students' view of science and dialectical ability, and influences the establishment of mathematical thinking, as well as causes negative function on improving students' mathematical application ability. ${ }^{[8]}$ In reality, large quantity of results obtained in mathematics subject are gradually produced due to its own demand, and those results are applied in later practice, including two kinds of different application types. However, we shall know the point that the college students' improvement of mathematical application ability can't rely on mathematical classroom teaching, and they mainly learn awareness and method of mathematical application in classroom. Meanwhile, in order to realize good effect, it is required to cultivate the mathematical application ability in whole science and engineering education of colleges.

\section{Conclusion}

In conclusion, the author thinks that the mathematical theory and method play a very important role in mathematics education; they are not the important precondition of mathematical education, but also the basic guarantee for realizing the objective of mathematical education. The systematic mathematical theory teaching shall be linked with mathematical application background and thoughts; otherwise it is not integral. The purpose of mathematical education is to make students learn mathematical theory and thinking, improve application ability, and improve their quality as a whole, and to improve their mathematical application ability is one of the most prominent purposes. However, no cultivation layer and one-sided mathematical pragmatism which lacks of cultivation objects will influence the exertion of mathematical education function to a great degree. Finally, the author thinks that the achievements that the scientific and technical personnel can obtain in long-time practice process can't be judged according to their learning situation in school days, and the college students shall always insist on learning mathematical theory and enhancing application ability. In this process, we shall establish management and evaluation standard for scientific and technical personnel in a more scientific and perfect way so as to realize the maximization of social benefit of college mathematical education.

\section{References}

[1] Cao Dingzhong, Li Danheng, Liu Chunzhong, etc.: Thought on Reform in Mathematical Teaching Methods, Means, Evaluation Content and Methods of Chinese Colleges, Mathematical Theory and Applications, 2011 (04): 28-29.

[2] Gao Chengxiu, Yi Xuming, Liu Guo, etc.: Teaching, Competition, and Innovative Education of Mathematical Modeling, Mathematics in Practice and Theory, 2011 (05): 623-624.

[3] Guo Xiaoli, Wang Xiangdong: Discussion on Implementation of Quality-oriented Education in Higher Mathematics Education, Mathematics in Practice and Theory, 2012 (06): 761-763.

[4] He Xiaoliang, Dai Yonghong, Zhou Yicang: Exploration on Mathematical Modeling Experiment, Mathematics in Practice and Theory, 2012 (05): 625-626.

[5] Zhu Hong, Wang Wei: Exploration and Practice on Evaluation Mode of College Mathematics Course, Experiment Science and Technology, 2011 (04): 108-111.

[6] Huang Zhiqin, Sun Hongwei: Several Attempts on Penetration Modeling Thought in Higher Mathematical Teaching, Mathematical Education, 2013 (03): 69-71.

[7] Jiang Qiyuan: Develop the Potential of Technical Transformation to Enhance the Strength of Economic Competition - application dynamics of mathematical modeling in the field of engineering technology, and economic management, Mathematics in Practice and Theory, 2012 (01): 80-87. 
[8] Li Shangzhi, Chen Fazhou: Knowledge and Practice of Mathematical Experiment Course Construction, Mathematics in Practice and Theory, 2011 (06): 764-768. 